\title{
Two cases of delayed diagnosis of postpartal streptococcal toxic shock syndrome
}

\author{
Wolfram Schummer and Claudia Schummer \\ Clinic for Anesthesiology and Intensive Care Medicine, Friedrich-Schiller University, Jena, Germany
}

\begin{abstract}
Background: Puerperal sepsis due to group A $\beta$-hemolytic streptococcal (GAS) toxic shock syndrome is associated with very high morbidity and mortality. Luckily it is now rare, but diagnosis is not always easy. This report demonstrates the problem of recognizing this disease, and summarizes the current knowledge on the pathomechanism and management of streptococcal toxic shock syndrome.

Case: Two cases of postpartum streptococcal toxic shock syndrome due to GAS are described. In both cases the correct diagnosis was delayed for several days. The first patient was sent home with the diagnosis of German measles; the second patient was transferred to our neurological intensive care unit with the diagnosis of meningitis. Both patients were admitted to the intensive care unit in profound shock, both developed multiple organ failure, and one patient died.

Conclusions: GAS may produce virulence factors that cause host tissue pathology. Besides aggressive modern intensive care treatment, early diagnosis and correct choice of anti-streptococcal antibiotics are crucial. A possible adverse effect of non-steroidal anti-inflammatory agents requires confirmation in a multicenter study.
\end{abstract}

Key words: Puerperal Sepsis; Streptococcal Infections; Bacterial Antigens; Non-Steroidal Anti-Inflammatory Agents; Adverse EfFects

\section{INTRODUCTION}

Puerperal sepsis is a preventable postpartal complication, if early signs are recognized and treated adequately. The most serious postpartal infection is the streptococcal toxic shock syndrome (TSS), characterized by the sudden onset of septic shock with multiple organ failure and the isolation of group A $\beta$-hemolytic streptococci (GAS) from a normally sterile body site. The dramatic nature of this severe, life-threatening infection is illustrated by the following two cases. Knowledge of the pathomechanism of invasive streptococcal infections might help to understand the course of this disease.

\section{CASE I}

After normal vaginal delivery of a healthy boy, the first patient (gravida two, para two) developed fever, combined with a macular rash especially of the trunk, 3 days postpartum. She was given non-steroidal anti-inflammatory drugs (NSAIDs) against her fever and was discharged home early with the diagnosis of German measles (Figure 1). Seven days postpartum she was readmitted with the features of septic shock: hypotension, tachycardia, tachypnea, and pyrexia. She was put on metronidazole in combination with mezlocillin. Despite treatment with fluids and vasoactive drugs under early invasive hemodynamic monitoring she

Correspondence to: Wolfram Schummer, MD, DEAA, EDIC, Clinic for Anesthesiology and Intensive Care Medicine,

Friedrich-Schiller University, Bachstr. 18, 07743 Jena, Germany. Email:cwsm.schummer@gmx.de 
progressed to organ dysfunction (Table 1). The abdomen was soft; the uterus was bulky, anteverted, and tender. Since the focus of infection was considered to be the uterus, a hysterectomy was performed. When the diagnosis of a Group A $\beta$-hemolytic streptococcal infection was confirmed from blood cultures and abdominal swabs both taken on the day of admission to the intensive care unit (ICU) (day 1) - penicillin G was added to

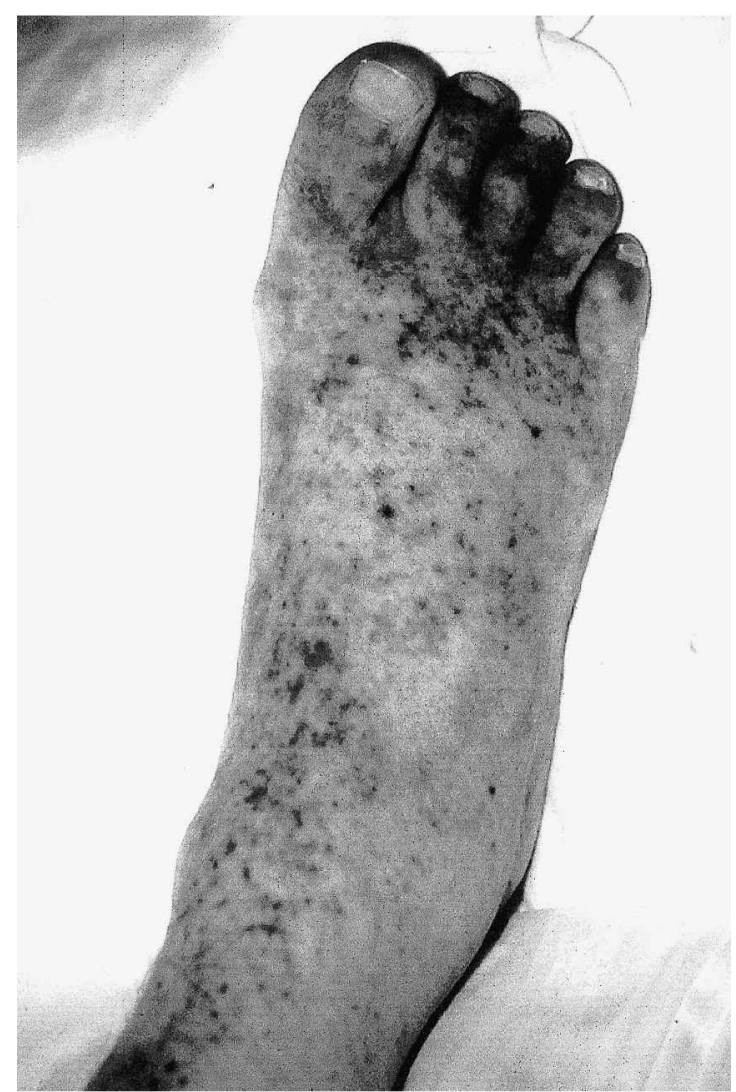

Figure I Patient I on readmission: petechial rash of right foot the antimicrobial regimen 24 hours later. This was done because the activity of Penicillin G against GAS is four times the activity of Mezlocillin. With regard to surgery, it was decided against a narrowing of antimicrobial therapy. Her stay in the ICU was initially characterized by septic shock, disseminated intravascular coagulation (DIC), and multiple organ failure, including acute renal failure with the need for continuous veno-venous hemofiltration. The severity and course of illness are depicted in Figures 2 and 3, respectively. Secondary, left ventricular decompensation due to post-streptococcal myocarditis and critical illness polyneuropathy characterized her further stay in the ICU. After 28 days, she returned to the ward prior to discharge home.

\section{CASE 2}

The second patient (gravida two, para one) was admitted to the labor ward with premature rupture of membranes in week 38 of gestation. She delivered vaginally a healthy girl within 24 hours after rupture of membranes. She did not receive any antimicrobial therapy. Three days later she complained of a frontal headache and muscle ache in all limbs combined with a fever up to $39.5^{\circ} \mathrm{C}$ that was treated initially with a NSAID. When she became stuperous another 3 days later she was transferred to a neurological ICU with the diagnosis of meningitis. On admission she was comatose, in severe shock and needed immediate intubation (Table 1). Aggressive organ support was initiated immediately. A computed cranial tomogram was normal, the cerebrospinal fluid was clear, she showed $158 / 3$ cells with an increase in granulocytes, $584 \mathrm{mg} / \mathrm{l}$ protein, and a massive

Table I Patient characteristics on admission (day I) and after I day of aggressive supportive intensive care therapy (day 2)

\begin{tabular}{lccccc}
\hline & \multicolumn{2}{c}{ Patient I } & \multicolumn{3}{c}{ Patient 2 } \\
\cline { 2 - 3 } \cline { 5 - 6 } Characteristics & Day I & Day 2 & & Day I & Day 2 \\
\hline Temperature $\left({ }^{\circ} \mathrm{C}\right)$ & 40.1 & 39 & & 40.4 & 39.4 \\
Pulse rate $(\mathrm{bpm})$ & 192 & 180 & 160 & 180 \\
Blood pressure $(\mathrm{mmHg})$ & $80 / 40$ & $100 / 50$ & & $70 / 30$ & $110 / 50$ \\
White blood cell count $(109 / \mathrm{l})$ & $8.9(29 \%$ bands) & 8.6 & & 21.7 & 12.4 \\
Urine output $(\mathrm{ml} / 6 \mathrm{~h})$ & 45 & 130 & & 70 & - \\
\hline
\end{tabular}


elevated lactate. For streptococcal TSS (puerperal sepsis) she was directly administered piperacillin, sulbactam, and clindamycin for 6 days. After 24 hours gram-positive, catalase-negative cocci were grown from two vaginal swabs and blood cultures confirmed the diagnosis. The colonies displayed a strong $\beta$-haemolysis; classification of the bacteria, which had been identified as Streptococcus pyogenes, detected Lancefield Group A antigen. Sequencing of the emm gene revealed type 77/27L. The genes for the exotoxins SPE C and SPE F could be detected by polymerase chain reaction. Multiple organ failure (cardiovascular, respiratory, renal) and DIC had already been established at admission to the ICU. Acute anuric renal failure indicated continuous venovenous hemofiltration from day 2 onwards. Severely compromised respiratory and cardiovascular function necessitated continuous therapeutic intervention. The severity and the course of illness are again demonstrated by Figures 2 and 3. Organ functions stabilized after 12 days but then, unfortunately, she had massive infratentorial bleeding which lead to brain death on day 16.

\section{DISCUSSION}

Puerperal infection due to GAS became a rare disease in the 20th century. In recent years GAS have been more important as a cause of perinatal maternal mortality, and in the last decade there has been again an increase of GAS infection ${ }^{1}$. The only prospective population-based surveillance of invasive GAS disease was performed in Canada in $1992-3^{2}$. Invasive GAS disease was defined by the isolation of $S$. pyogenes from a normally sterile body site. The annual incidence of GAS was 1.5 cases

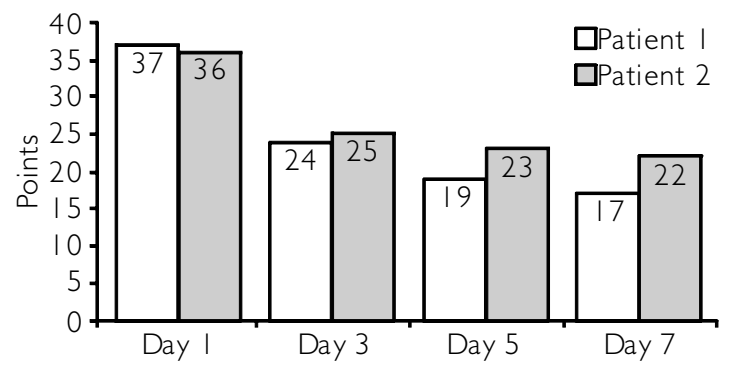

Figure 2 Course of Acute Physiology and Chronic Health Evaluation (APACHE) II scores per 100000 population; the rate for puerperal GAS sepsis was 0.5 infections per 10000 deliveries; and 0.2 GAS infections per 10000 met the criteria for streptococcal toxic shock syndrome (streptococcal TSS) (Table 2) ${ }^{3}$. Here the overall mortality rate was $81 \%$.

As is shown by our cases, early signs and symptoms of puerperal sepsis due to GAS are apparently untypical and are often misinterpreted due to the low incidence of this fulminating disease (Table 3). Both patients received NSAIDs against their unspecific symptoms. In case 1 the diagnosis was made 4 days after the occurrence of an erythema and in case 2, 3 days after the onset of the first symptoms. In our two cases, diagnosis of GAS was

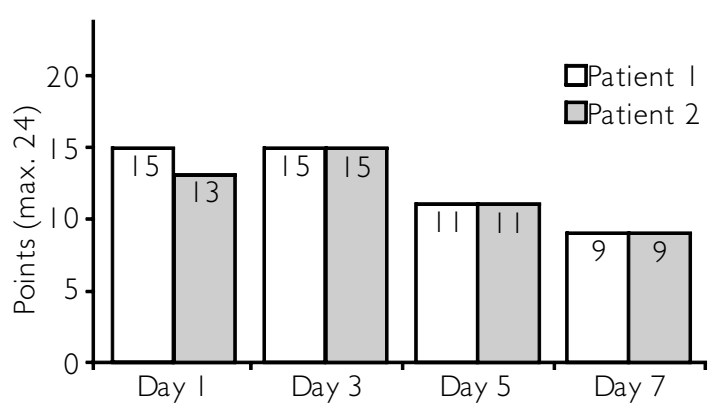

Figure 3 Course of Multiple Organ Dysfunction Scores (MODS)

Table 2 Streptococcal toxic shock syndrome is defined as any group A streptococcal infection associated with the early onset of shock and organ failure ${ }^{15}$

A Isolation of group A streptococcus

(I) From a sterile site

(2) From a non-sterile body site

B Clinical signs of severity

(I) Hypotension

(2) Clinical and laboratory abnormalities (requires two or more of the following:

(a) Renal impairment

(b) Coagulopathy

(c) Liver abnormalities

(d) Acute respiratory distress syndrome

(e) Extensive tissue necrosis, i.e. necrotizing fasciitis

(f) Erythematous rash)

Definite case: $\mathrm{AI}+\mathrm{B}(\mathrm{I}+2)$

Probable case: $\mathrm{A} 2+\mathrm{B}(\mathrm{I}+2)$ 
confirmed by blood cultures and abdominal and vaginal swabs. At that time both patients suffered already from septic shock according to ACCP/ SCCM criteria ${ }^{4}$ and multiple organ failure. To demonstrate the severity and the course of illness and organ dysfunction we applied the Acute Physiology and Chronic Health Evaluation (APACHE) II and the Multiple Organ Dysfunction Score (MODS) (Figures 2 and 3). The APACHE II model uses information on physiological variables, age, surgical status, and previous health status to assign a score to the patient

Table 3 Early clinical signs suggesting puerperal sepsis

Clinical signs suggestive of puerperal sepsis

Pains: symphyseal, limbs, joints, sore throat, headache

Malaise, flu-like symptoms

Short fever, or none

Erythematous rash

Obstipation, diarrhoea

Acral mottling

Altered mental state

Tachycardia
(Table 4). The APACHE II is the most commonly used outcome prediction model ${ }^{5}$. The organ failure scores were developed to quantify organ failure in critically ill patients. A commonly used score is the Multiple Organ Dysfunction Score (MODS). This sophisticated score describes increasing dysfunction of individual organs, measured as a continuum ranging from normality through dysfunction to complete failure ${ }^{6}$.

GAS may produce virulence factors that cause host tissue pathology. These virulence factors have been grouped into four families: adhesins, aggressins (i.e. streptolysin O), impedins (i.e. superantigens), and invasins. The streptococcal pyrogenic exotoxins (SPEs) are polypeptides, produced by certain group A streptococci and belong to the group of substances known as superantigens ${ }^{7}$. They have the capacity to superstimulate the immune system and thereby induce a cascade of cytokines. The action of cytokines is assumed to be responsible for the induction of the TSS. Superantigens such as the streptococcal pyrogenic exotoxins do not require processing by antigen-presenting cells before they can stimulate T-cells. These superantigens cause an aberrant

Table 4 The APACHE II is the most commonly used outcome prediction model. Acute Physiology Score (APS), the largest component of the APACHE II score, is derived from 12 clinical measurements that are obtained within 24 hours after admission on the ICU. The most abnormal measurement is selected to generate the APS component ${ }^{5}$

\begin{tabular}{|c|c|c|c|c|c|c|c|c|c|}
\hline APS/points & +4 & +3 & +2 & +1 & 0 & +1 & +2 & +3 & +4 \\
\hline Temperature $\left({ }^{\circ} \mathrm{C}\right)$ & $\geq 4$ I & $39-40.9$ & & $38.5-38.9$ & $36-38.4$ & $34-35.9$ & $32-33.9$ & $30-31.9$ & $\leq 29.9$ \\
\hline $\begin{array}{l}\text { Mean arterial pressure } \\
\quad(\mathrm{mmHg})\end{array}$ & $\geq 160$ & $130-159$ & $110-129$ & & $70-109$ & & $50-69$ & & $\leq 49.9$ \\
\hline Heart rate $(\mathrm{bpm})$ & $\geq 180$ & $140-179$ & $110-139$ & & $70-109$ & & $55-69$ & $40-54$ & $\leq 39$ \\
\hline $\begin{array}{l}\text { Respiratory rate } \\
\text { (per minute) }\end{array}$ & $\geq 50$ & $35-49$ & & $25-34$ & $12-24$ & $10-11$ & $6-9$ & & $\leq 5$ \\
\hline $\begin{array}{l}\text { Oxygenation: if } \mathrm{FiO}_{2} \geq \\
0,5 \mathrm{~A}: \mathrm{AaDO}_{2}\end{array}$ & $\geq 500$ & $350-499$ & $200-349$ & & $<200$ & & & & \\
\hline $\begin{array}{l}\mathrm{FiO}_{2}<0,5: \mathrm{PaO}_{2} \\
\quad(\mathrm{mmHg})\end{array}$ & & & & & $>70$ & $6 I-70$ & & $55-60$ & $<55$ \\
\hline Arterial $\mathrm{pH}$ & $\geq 7.7$ & $7.6-7.69$ & & $7.5-7.59$ & $7.33-7.49$ & & $7.25-7.32$ & $7.15-7.24$ & $<7.15$ \\
\hline $\mathrm{Na}^{+}(\mathrm{mmol} / \mathrm{l})$ & $\geq 180$ & $160-179$ & $155-159$ & $150-154$ & $130-149$ & & $120-129$ & $111-119$ & $\leq 110$ \\
\hline $\mathrm{K}^{+}(\mathrm{mmol} / \mathrm{l})$ & $\geq 7$ & $6-6.9$ & & $5.5-5.9$ & $3.5-5.4$ & $3.3-3.4$ & $2.5-2.9$ & & $<2.5$ \\
\hline $\begin{array}{l}\text { Creatinine }(\mathrm{mg} / \mathrm{dl}) \\
\quad(\text { acute renal failure: } \times 2)\end{array}$ & $\geq 3.5$ & $2-3.4$ & $1.5-1.9$ & & $1.6-1.4$ & & $<0.6$ & & \\
\hline Hematocrit (\%) & $\geq 60$ & & $50-59.9$ & $46-49.9$ & $30-45.9$ & & $20-29.9$ & & $<20$ \\
\hline White blood cells $\left(10^{9} / \mathrm{I}\right)$ & $\geq 40$ & & $20-39.9$ & $15-19.9$ & $3-14.9$ & & $1-2.9$ & & $<1$ \\
\hline I5 - Glascow Coma Scale & & & & & & & & & \\
\hline
\end{tabular}


proliferation of specific $\mathrm{T}$-cell subsets and can directly activate thousands of times more T-cells (up to $25 \%$ of the total $\mathrm{T}$-cell population) than conventional antigens, to produce an accelerated cascade of cytokines such as interleukins and tumor necrosis factor $(\mathrm{TNF})^{8}$. At this stage, antibiotics alone can't control GAS infection.

Additionally we would like to report on an observation concerning NSAIDs. We have treated seven patients with proven GAS-TSS either as puerperal sepsis or as necrotizing fasciitis. All patients had ingested NSAIDs while showing early signs of their illness. For us it is suggestive that NSAIDs taken at the early stage of invasive GAS infection may accelerate the cascade systems and play a contributory role in the onset of shock, organ failure, cytokine production, and inflammation ${ }^{9}$. This observation would require confirmation in a multicenter study.

Other streptococcal factors, such as streptolysin $\mathrm{O}$, contribute to production of TSS because this substance can, at least in vitro, induce TNF and seems to function synergistically with exotoxin ${ }^{10}$. Due to their virulence factors, GAS spread rapidly and stimulate the cascade systems maximally ${ }^{11}$. The uterus might be the site of initial infection but after the rapid hematological spread the uterus is no longer considered the focus of infection. Therefore currently hysterectomy is not part of first-line treatment. With streptococal TSS due to puerperal sepsis a patient should be stabilized within 24-48 hours. If a patient's condition deteriorates despite adequate intensive care therapy (antibiotics: penicillin $G$ and clindamycin, and support of failing organs), hysterectomy should be performed ${ }^{12}$.

The therapeutic management of severe GAS infections requires high doses of intravenous antibiotics. Penicillin $G$ traditionally has been the agent of choice, because there is no high-level resistance among the group A streptococci. Patients allergic to penicillin may be treated with a first-generation cephalosporin, vancomycin, or clindamycin. Recently, clindamycin has been recommended in addition to penicillin for all patients. Experimental evidence indicates that clindamycin may be more effective than penicillin at inhibiting these organisms when they are present in a high inoculum and not dividing rapidly. Evidence comes from in vitro observation of the 'Eagle effect' as well as animal models. Harry Eagle first showed that streptococci present at high inoculum $\left(>10^{7} \mathrm{cfu} / \mathrm{mL}\right)$ are very slowly killed by penicillin. Animal models suggest that clindamycin, with its inhibition of protein synthesis, has greater activity than penicillin against organisms in a stationary phase of growth and may reduce production of toxins, and $\mathrm{TNF}^{13}$. Clindamycin alone is not recommended because some strains are resistant, especially in regions where macrolides are used extensively in outpatients. Invasive streptococcal TSS is fulminating and may not respond to delayed antibiotic therapy; therefore it is obvious that antibiotics and aggressive intensive care treatment should be started as early as possible.

Immunoglobulin preparations have been given to patients with the intention of neutralizing the streptococcal toxins, but none of the reports on this practice is conclusive ${ }^{14}$. However, ordinary human immunoglobulin preparations contain considerable amounts of toxin-neutralizing antibodies and, given at an early stage of invasive disease, might have a beneficial effect ${ }^{15}$. No human studies on intravenous immunoglobulins for streptococcal TSS have been performed so far. And

Table 5 The Multiple Organ Dysfunction Score (MODS); PAR, pulse-adjusted heart rate = HR $\times(R A P / M A P) ; H R$, heart rate; RAP, right atrial pressure; MAP, mean arterial pressure; *the best estimate in the absence of sedation ${ }^{6}$

\begin{tabular}{|c|c|c|c|c|c|}
\hline Characteristic/points & 0 & I & 2 & 3 & 4 \\
\hline $\mathrm{PaO}_{2} / \mathrm{FiO}_{2}$ & $>300$ & $226-300$ & $|5|-225$ & $76-150$ & $<76$ \\
\hline Serum creatinine $(\mathrm{mg} / \mathrm{dl})$ & $<1.1$ & $1.11-2.29$ & $2.3-3.99$ & $4-5.7$ & $>5.7$ \\
\hline Serum bilirubin $(\mathrm{mg} / \mathrm{dl})$ & $<1.19$ & $1.2-3.5$ & $3.5 \mathrm{I}-7$ & $7.01-14$ & $>14$ \\
\hline PAR & $<10$ & $10.1-15$ & $15.1-20$ & $20.1-30$ & $>30$ \\
\hline Platelet count (per $\mu \mathrm{L}$ ) & $>120$ & $81-120$ & $5 \mathrm{I}-80$ & $21-50$ & $<21$ \\
\hline Glascow Coma Score* & 15 & $13-14$ & $10-12$ & 7-9 & $<7$ \\
\hline
\end{tabular}


there is still no evidence for the use of other experimental approaches like the application of anti-TNF and plasmapheresis, respectively.

Conclusion: currently, an otherwise healthy patient, with rapid onset of shock and fever even after an otherwise normal delivery is at least likely to suffer from streptococcal TSS. In the absence of effective methods of prevention of this devastating disease, a high index of suspicion, prompt initiation of antibiotic therapy with agents that suppress toxin production, and aggressive organsupporting intensive care therapy constitute our best weapons to fight TSS.

\section{ACKNOWLEDGEMENTS}

The authors are grateful to Dr. A. Weiss for helpful suggestions and review of this work. The authors also would like to acknowledge all of the staff of the ICU who were involved in the care of these patients. We thank Dr. D. Mack, Institut für Medizinische Mikrobiologie und Immunologie, Universitätsklinikum Hamburg-Eppendorf and Dr. C. Brandt, Institut für Medizinische Mikrobiologie, Universitätsklinikum der RheinischWestfälischen Technischen Hochschule Aachen, for the isolation and biochemical identification of the $S$. pyogenes strain of the second patient.

\section{REFERENCES}

1. Stevens DL. Invasive group A streptococcus infections. Clin Infect Dis 1992;14:2-11

2. Davies HD, McGeer A, Schwartz B, et al. Invasive group A streptococcal infections in Ontario, Canada. Ontario Group A Streptococcal Study Group. N Engl J Med 1996;335:547-54

3. Infection TwGoSS. Defining the group A streptococcal toxic shock syndrome. Rationale and consensus definition. The Working Group on Severe Streptococcal Infections. J Am Med Assoc 1993; 269:390-1

4. Bone RC, Sibbald WJ, Sprung CL. The ACCPSCCM consensus conference on sepsis and organ failure. Chest 1992;101:1481-3

5. Knaus WA, Draper EA, Wagner DP, et al. APACHE II: a severity of disease classification system. Crit Care Med 1985;13:818-29

6. Marshall JC, Cook DJ, Christou NV, et al. Multiple organ dysfunction score: a reliable descriptor of a complex clinical outcome. Crit Care Med 1995; 23:1638-52

7. Bohach GA, Fast DJ, Nelson RD, et al. Staphylococcal and streptococcal pyrogenic toxins involved in toxic shock syndrome and related illnesses. Crit Rev Microbiol 1990;17:251-72

8. Schlievert PM. Role of superantigens in human disease. J Infect Dis 1993;167:997-1002

9. Stevens DL. Could nonsteroidal antiinflammatory drugs (NSAIDs) enhance the progression of bacterial infections to toxic shock syndrome? Clin Infect Dis 1995;21:977-80

10. Hackett SP, Stevens DL. Streptococcal toxic shock syndrome: synthesis of tumor necrosis factor and interleukin-1 by monocytes stimulated with pyrogenic exotoxin A and streptolysin O. J Infect Dis 1992;165:879-85

11. Sriskandan S, Kemball-Cook G, Moyes D, et al. Contact activation in shock caused by invasive group A Streptococcus pyogenes. Crit Care Med 2000;28:3684-91

12. Petersen EE. Streptokokken-A-Puerperalsepsis. Der Gynäkologe 1999;32:512-17

13. Stevens DL, Gibbons AE, Bergstrom R, et al. The Eagle effect revisited: efficacy of clindamycin, erythromycin, and penicillin in the treatment of streptococcal myositis. J Infect Dis 1988;158:23-8

14. Stevens DL. Rationale for the use of intravenous gamma globulin in the treatment of streptococcal toxic shock syndrome. Clin Infect Dis 1998;26: 639-41

15. Stegmayr B, Bjorck S, Holm S, et al. Septic shock induced by group A streptococcal infection: clinical and therapeutic aspects. Scand J Infect Dis 1992;24:589-97

16. Stevens DL. Invasive group A streptococcal infections: the past, present and future. Pediatr Infect Dis J 1994;13:561-6 


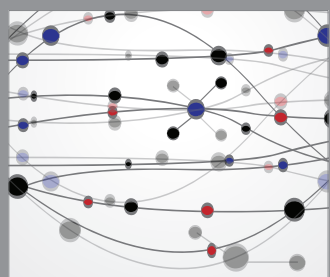

The Scientific World Journal
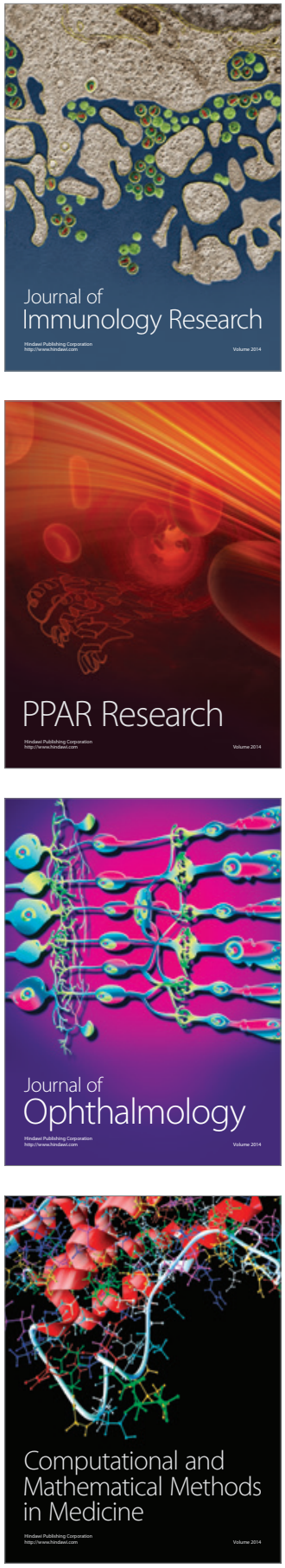

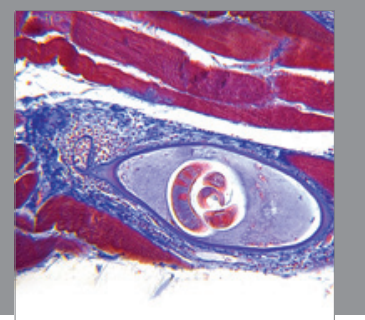

Gastroenterology

Research and Practice
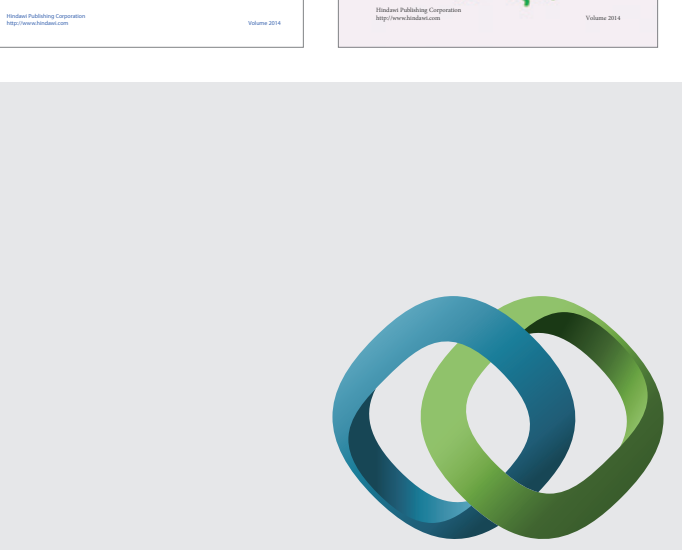

\section{Hindawi}

Submit your manuscripts at

http://www.hindawi.com
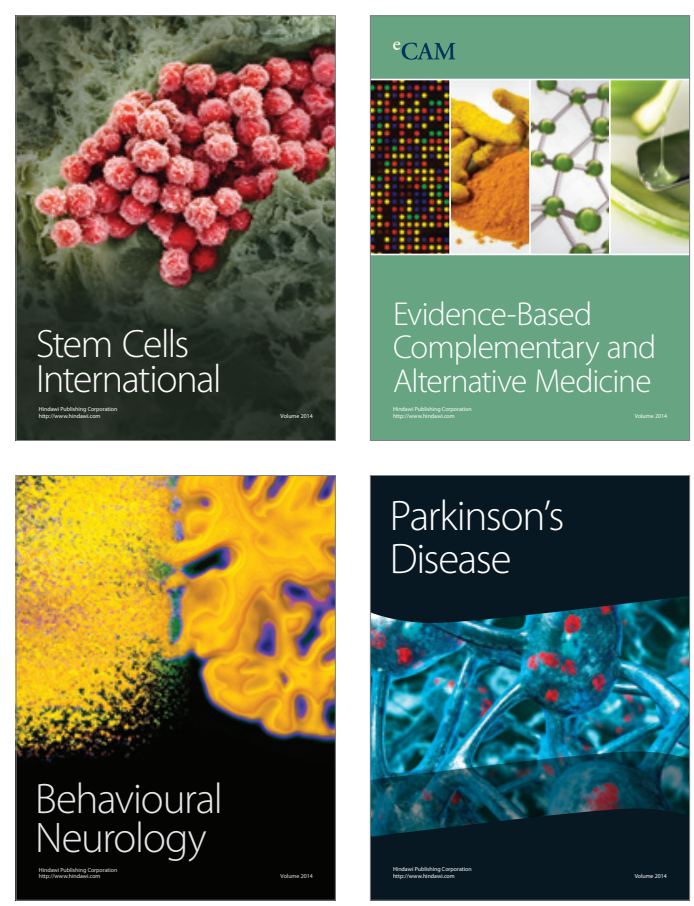

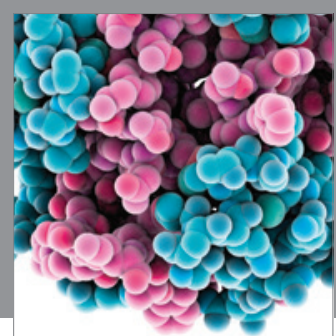

Journal of
Diabetes Research

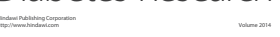

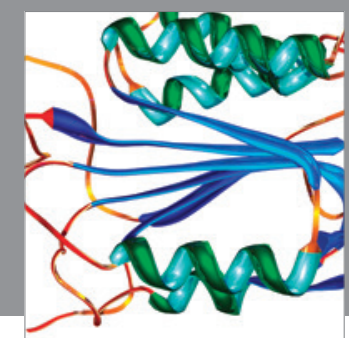

Disease Markers
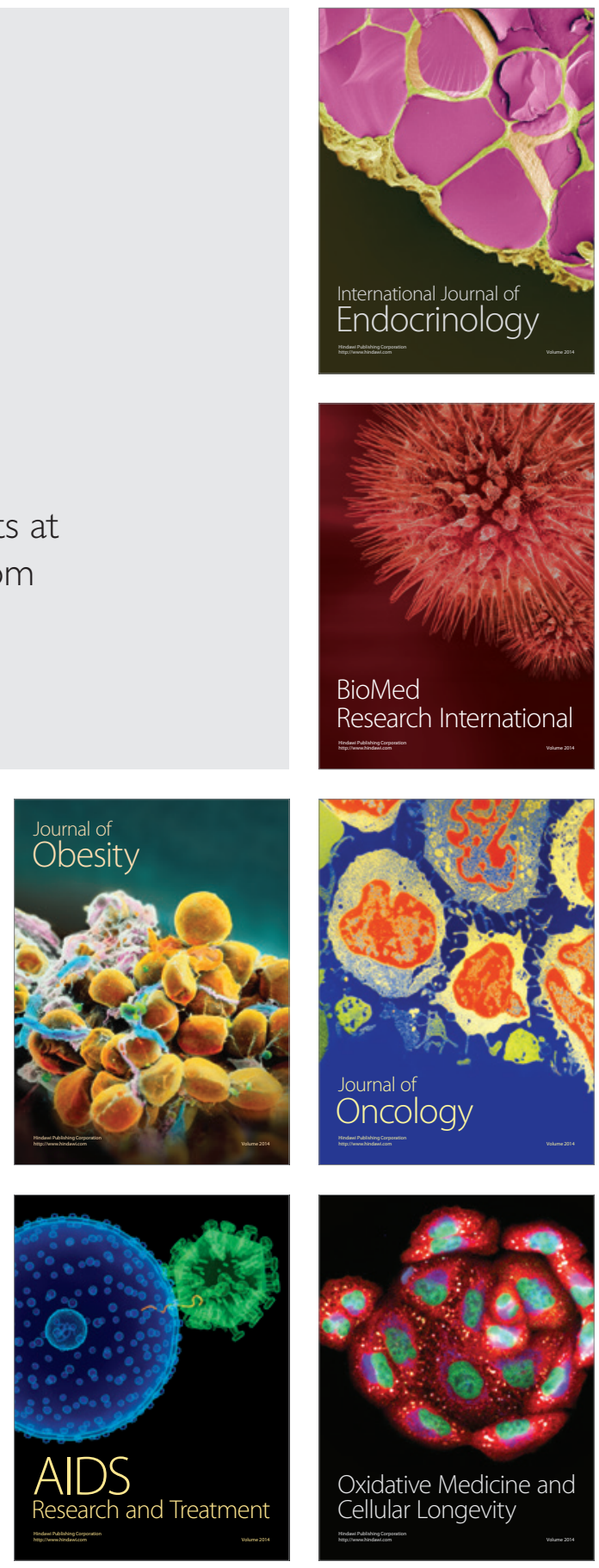